\title{
Hierarchical Sparsity-Regularized Framework Based Frequency Hopping Spectrum Estimation With Antenna Array System
}

\author{
Lifan Zhao, Lu Wang, Bi Guoan \\ School of Electrical and Electronic Engineering \\ Nanyang Technological University \\ Singapore \\ \{zhao0145,wa0001lu,egbi\}@ntu.edu.sg \\ Haijian Zhang \\ School of Electronic Information \\ Wuhan University \\ Wuhan, China \\ haijian.zhang@whu.edu.cn
}

\begin{abstract}
Frequency hopping (FH) signals have been widely employed in wireless communication networks to combat interference and avoid collision. This paper considers the blind FH signal estimation problem in antenna array systems, where the direction-ofarrivals, hopping time and frequency are all unknown to the users. A hierarchical sparsity-aware technique is developed to estimate these parameters in an optimization framework. More concretely, sparsity in spatial domain and time-frequency domain are exploited in a hierarchical and iterative manner, respectively, where more accurate parameter estimation performance can be obtained. Compared to prior state-of-the-arts, conventional model-order selection procedure can be conveniently avoided due to the utilization of sparsity-regularized framework. Results of numerical experiments show that the proposed algorithm can achieve superior performance particularly in sub-Nyquist sampling and low signal-to-noise ratio (SNR) scenarios compared with other recently reported ones.
\end{abstract}

Keywords-Frequency hopping, antenna array systems, sparsity-regularized framework

\section{INTRODUCTION}

In wireless communications and other information systems, frequency hopping (FH) signals have been widely employed due to their advantageous capability of low interception and anti-jamming [1]-[3]. In many wireless networks such as home area networks (HAN) and Blue tooth personal area network(PAN), FH signal is employed to combat near-far problem[4] and avoid collision [1], [5], [6]. When non-cooperative FH networks coexist, however, it is inevitable that the receiver encounters multiple unknown $\mathrm{FH}$ signals from different emitters. In these systems, antenna array is often utilized to provide spatial degree of freedom to separate sources from different directions. The main challenge is to robustly estimate the DOAs, hopping time and frequency of the multiple FH signals. Since the maximum likelihood estimation will induce intractable computations and cause over-fitting problems [7],[8], many other alternative approaches are developed to achieve robust estimation.

The conventional time-frequency distribution (TFD) is a natural tool to exhibit the non-stationary frequency content of the signals. DOA estimation procedure, such as beamforming or multiple signal classification (MUSIC), is often carried out before applying TFD to estimate the hopping time and frequency in a non-parametric manner. The linear TF distribution such as, short-time Fourier transform (STFT), is a popular choice for estimating the parameters of each FH signal due to it is free of cross-terms [9], while quadratic TFD such as, Wigner-Ville distribution (WVD) is also employed for its high energy concentration of the signal [10]. Even though entropy or gradient based refinement is often employed to the TFD as a post-processing procedure, either limited resolution (linear TFD) or undesirable cross-terms (quadratic TFD) greatly inhibit substantial improvements by these procedures. In [1], [11], another method is developed to cope with DOA, hopping time and frequency estimation in FH networks. An expectation maximization (EM) approach is employed to iteratively estimate the amplitude, hopping time and frequency. In this approach, the success of the EM algorithm depends largely on the proper selection of initial value, where a denoised STFT is often used to initialize the algorithm. In low SNR environments, however, it is hard to obtain a desirable initial TFR, which will inevitably result in degraded 
performance of the EM algorithm. More importantly, the model order selection procedure is required to facilitate the estimation. To effectively deal with the heavy burden on A/D hardware, multiple FH signal estimation is considered in a sparse linear regression (SLR) framework [8], [12], where a fused-lasso alike formulation is employed to solve this problem. In this framework, two penalty terms are defined to encourage sparsity [13] and smoothness in the TF domain, respectively. The formulation can be expressed as,

$$
\hat{\mathbf{x}}=\arg \min _{\mathbf{x}}\left[\frac{1}{2}\|\mathbf{y}-\mathbf{W} \mathbf{x}\|_{2}^{2}+\lambda_{1}\|\mathbf{x}\|_{1}+\lambda_{2}\|\mathbf{D} \mathbf{x}\|_{1}\right]
$$

where the first term is for data fitting, the second and third terms are for encouraging sparsity in frequency and differential-time domain, respectively [8]. One remarkable advantage of this approach is that it does not require to select the order of the parameters. Moreover, due to the grid based formulation, this approach can even be conveniently accommodate to different sampling strategy [8]. However, this approach is particularly formulated for single channel system, where non-trivial modification is required to be carried out for multi-channel systems. Inspired by the SLR framework, we propose a hierarchical sparsity-regularized framework to cope with the abovementioned challenges. In the first stage of the framework, spatial sparsity of the signals is exploited, while frequency and hopping sparsity are exploited in the second stage of the framework. The main idea of the algorithm is to properly utilize the hierarchical sparsity model to achieve jointly sparsity. By unifying these two stages in an iterative estimation, more accurate estimation performances can be expected. The proposed method can be considered as a modelselection free approach, where model-order mismatch problem is desirably avoided by the utilization of sparsity. Moreover, the proposed approach can allow for mutual improvements of parameter estimation due to the proposed iterative scheme.

The paper is organized as follows. In Section II, the preliminary is presented and the hierarchical sparse model is introduced. In Section III, the sparsity-regularized framework is formulated in a hierarchical manner. The proposed approach is proposed in Section IV of the paper. Finally, the experimental results are given in Section $\mathrm{V}$ to validate the performance of the proposed algorithms.

Notation : Vectors and matrices are denoted by bold symbol. For a vector $\mathbf{x}, \mathbf{x}^{*}$ and $\mathbf{x}^{H}$ represent the conjugate and Hermite of the vector. For a matrix $\mathbf{A}, \mathbf{A}^{H}$ and $\mathbf{A}^{-1}$ denote the conjugate and inverse of the matrix, respectively. $l_{p}$ norm of the vector or matrix is denoted by $\|\cdot\|_{p} . C N(\boldsymbol{X} \mid \mu ; \Sigma)$ denotes multivariate complex Gaussian distributions.

\section{PRELIMITARY AND PROBLEM FORLUMATION}

In the multi-channel FH sensor network, the direction-of arrival (DOA), hopping time and frequency are all required to be estimated by each user. In particular, the geometry of the uniform linear array (ULA) is given in Fig. 1. In this section, we present the received signal model of the multiple $\mathrm{FH}$ signals. Assuming $K_{s}$ sources are impinging on a ULA with $L$ sensors, the received signal in each snapshot $n$ is given as

$$
\mathbf{y}(n)=\Phi \mathbf{s}(n)+\mathbf{v}(n), n=1, \ldots, N_{s}
$$

where $\mathbf{y}(n) \in \mathrm{C}^{L \times 1}, \Phi=\left[\mathbf{a}\left(\theta_{1}\right) ; \ldots, \mathbf{a}\left(\theta_{N 0}\right)\right] \in \mathrm{C}^{L \times N 0}, \mathbf{s}(n) \in$ $\mathrm{C}^{N 0 \times 1}$ contains the $\mathrm{FH}$ signals, $N_{s}$ is the number of snapshots. The noise $\mathbf{v}(n)$ is assumed to be spatially and temporally uncorrelated,

$$
\mathbb{E}\left[\mathbf{v}\left(n_{1}\right) \mathbf{v}^{H}\left(n_{2}\right)\right]=\alpha_{0}^{-1} \mathbf{I} \cdot \delta_{n_{1}, n_{2}} .
$$

Consider a FH network where each user receives the noise corrupted multiple FH signals. Sampling the continuous signal $\mathbf{s}(t)$, we can obtain the discrete form of $\mathbf{s}(t)$ expressed as

$$
\mathbf{s}(n)=\sum_{k=1}^{K_{i}} \sigma_{i, k} e^{j 2 \pi f_{i, k} n / f_{s}}, \quad n_{i-1}<n<n_{i}
$$

where $K_{i}$ is the number of hopping frequencies during the $i$ th system dwell time, $\sigma_{i ; k}$ is the amplitude of the signal associated with the $i$ th system dwell time and $k$-th hopping frequency. Since the noise obeys complex Gaussian distribution, the likelihood of the received signal can be expressed as

$$
p(\mathbf{Y} \mid \mathbf{S})=\prod_{k=1}^{N_{s}} \mathcal{C N}\left(\mathbf{y}(k) \mid \Phi \mathbf{s}(k), \sigma^{2}\right)
$$

In practical communication systems, direction of arrivals, the system dwell time and hopping frequency are all unknown to the user, and required to be estimated robustly to avoid retransmission. To illustrate the difficulty of the problem, the maximum likelihood (ML) estimation can be formulated as the following optimization problem given as,

$\left(\hat{\mathbf{n}}_{\mathbf{k}}, \hat{\mathrm{K}}, \hat{\mathrm{f}}, \hat{\mathrm{N}}_{k}, \hat{\sigma}\right)=\arg \min _{\hat{\mathbf{n}}_{\mathbf{k}}, \hat{\mathrm{K}}, \hat{\mathbf{f}}, \hat{\mathrm{N}}, \hat{\sigma}} \sum_{k=1}^{\hat{\mathrm{K}}}\left\|\mathbf{Y}_{k}-\sum_{n=1}^{\hat{N}_{k}} \hat{\sigma}_{k n} B\left(\hat{\mathrm{f}}_{k n}\right)\right\|_{F}^{2}(6)$

In blind frequency hopping signal estimation problem dealt within this paper, the frequency location, hopping time instant, the number of components are all unknown, which is a very challenging problem [8]. In conventional estimation scheme, model order should be selected for good performances. The above formulation is known to be a non-convex optimization problem and is analytically non-solvable [11], which is equivalent to the ML estimation. Since the hopping instant $n_{k}$, the number of hopping instants $\hat{K}$, the hopping frequency $f_{i, k}$, the number of components $N$, complex magnitude $\sigma_{k n}$ and even direction of arrivals $\theta_{k}$ (in multi-channel case) are all unknown, solving this problem by exhaustive search is computational intractable. It is argued that the ML estimation by searching are these parameters in a combinational way is not a trivial task [1], [8]. Moreover, proper model-order selection procedure is also required to be carried out to avoid over-fitting problem.

\section{HIERACHICAL SPARSITY-REGULARIZED FRAMEWORK}

Inspired by the sparsity regularized framework, in this section, a hierarchical framework is introduced to estimate the 
DOA and FH signal in a sparsity-driven manner. As presented in Section II, the estimation problem can be expressed in a hierarchical linear model. In sensor array system, the sparsity of the signals in spatial domain and time-frequency domain can be exploited, respectively. More concretely, the signals are only coming from a small number of angles, and can be represented in the time-frequency domain in a piecewise sparse manner. Remarkably, this sparse driven approach can

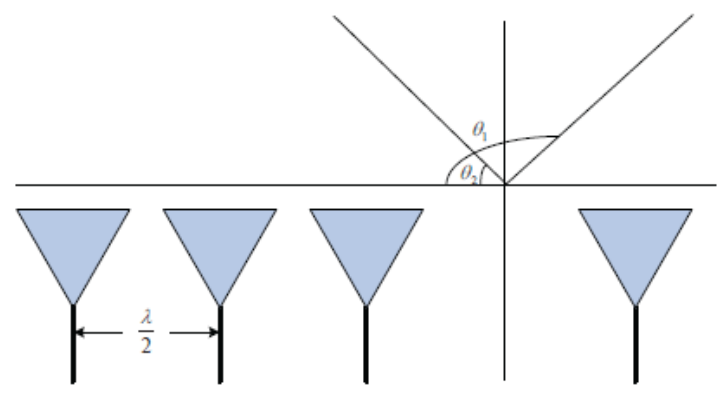

Fig. 1. The geometry of the ULA with two sources coming from $\theta_{1}$ and $\theta_{2}$. The antenna interval is set as $\lambda / 2$.

obtain desirable estimation results and avoid the model-order selection procedure.

\section{A. Stage 1: Spatial Sparsity}

To estimate the DOA with sparsity constraint [14], [15], a new matrix $\tilde{\mathbf{A}} \triangleq\left[\mathbf{a}\left(\theta_{1}\right), \mathbf{a}\left(\theta_{2}\right), \ldots, \mathbf{a}\left(\theta_{N_{0}}\right)\right] \in \mathbb{C}^{L \times N_{0}}$ is particularly manipulated as an over-complete dictionary, whose $i$-th atom

$$
\mathbf{a}\left(\theta_{i}\right)=\left[\mathrm{e}^{j \pi \cos \theta_{i}}, \mathrm{e}^{j 2 \pi \cos \theta_{i}}, \ldots, \mathrm{e}^{j L \pi \cos \theta_{i}}\right]^{H}
$$

corresponds to an ideal steering vector from spatial angle $\theta_{i}$, based on the geometry of the ULA presented in Fig. 1. Based on the sparse representation, the signal model can be constructed as,

$$
\mathbf{Y}=\tilde{\mathrm{A}} \mathbf{S}
$$

where $\mathbf{S}$ is a row-sparse matrix. The non-zero rows of the matrix $\mathbf{S}$ represent the FH signals from different directions. In this stage, the prior for the row-sparse matrix $\mathbf{S}$ can be expressed as,

$$
\mathrm{p}(\mathbf{S}) \propto \exp \left(-\lambda \sum_{k=1}^{N_{0}}\left|\sqrt{\sum_{i=1}^{N} x_{k i}^{2}}\right|\right)
$$

where $l$ is defined as a hyper-parameter to control the rowwise sparsity degree of the matrix $\mathbf{S}$. In particular, a non-zero row of the matrix $\mathbf{S}$ corresponds a $\mathrm{FH}$ signal from a particular direction.

\section{B. Stage 2: Hopping Time And Frequency Sparsity}

Apart from the spatial sparsity of the received signal, the FH signal exhibits hopping time and frequency sparsity in time-frequency domain in the modeling of the second stage
[16]. Let us denote the non-zero row of $\mathbf{S}$ as $\sim \mathbf{S}_{i}$. To exploit the hopping time and frequency sparsity, the signal model can be represented by,

$$
\mathbf{S}_{i}^{T}=\mathrm{Wx}_{i} \text {. }
$$

where $\mathbf{x}_{i} \in C^{N s N \times 1}$ is the stacked time-frequency coefficients for the $i$-th $\mathrm{FH}$ signal and $\mathbf{S}_{i} \in C^{1 \times N s}$ is the FHsignal from the $i$ th direction. The $i$-th row of matrix $\mathbf{W}$ is constructed as $\mathbf{W}_{i}=$ $\left[0_{N}^{H}, \ldots, 0_{N}^{H}, \omega^{T}, 0_{N}^{H}, \ldots, 0_{N}^{H}\right]^{T}$ where $\boldsymbol{\omega}=\left[e^{j \omega_{1} n}, \ldots, e^{j \omega_{N} n}\right]^{T}$ is a vector representing frequency grid. To exploit the frequency sparsity, the prior for $\mathbf{x} i$ is also a multi-variate Laplace distribution, expressed as [17]

$$
p_{1}\left(\mathrm{x}_{i}\right) \propto \exp \left(-\lambda_{1}\left\|\mathrm{x}_{i}\right\|_{1}\right)
$$

where the matrix $\mathbf{D}$ is a differential matrix defined in [8], [12]. Combining these two priors will result in both the hopping time and frequency prior for $\mathbf{x}_{i}$.

Based on the above described can be expressed as maximum a posterior (MAP) estimation, the solution of can be expressed as

$$
\begin{array}{r}
(\mathbf{S}, \mathbf{x})=\arg \max _{\mathbf{S}, \mathbf{x}} p(\mathbf{Y} \mid \mathbf{S}) p(\mathbf{S}) p_{1}(\mathbf{x}) p_{2}(\mathbf{x}) \\
\text { s.t. } \quad \mathbf{S}_{i}^{T}=\mathbf{W x}_{i}
\end{array}
$$

However, this MAP estimation problem is a hard to be solved directly due to the explicit coupling of prior for $\mathbf{S}$ and $\mathbf{x}$. Therefore, we propose an approximate algorithm to solve this problem in Section IV.

Remark 1: The above-describe model will naturally incorporate the sparsity in spatial, hopping time and frequency domain in a hierarchical manner. However, how to properly and effectively exploit this hierarchical sparsity to obtain mutual enhancement of DOA and FH signal estimation is the key issue. More concretely, exploiting the sparsity in the spatial domain should enhance the FH signal estimation performance and vice versa.

\section{FH SignAL ESTIMATION PROBLEM}

In this section, a hierarchical sparse regularized estimation (HSRE) approach is developed to obtain DOA and FH signal estimation in a joint manner. By leveraging the hierarchical sparsity, the immediate advantage of the proposed approach is

that it can desirably avoid model-order selection procedure. In particularly, an iterative procedure is proposed to estimate the DOA and FH signals in a hierarchical way. Another remarkable advantage of this approach is that it can achieve much better results by iteratively refining the DOA estimation and FH signal estimation. In this approach, the DOA estimation and FH signal estimation is carried out in separate stages, while allowing mutual improvement of the parameter estimation accuracy.

DOA estimation Stage: Assume that the FH signal has been estimated from the last iteration. To properly utilize the information, we propose to carry our the beam-forming procedure for the $i$-th FH signal, which can be expressed as, 


$$
\begin{aligned}
\tilde{\mathbf{Y}}_{1}^{i} & =\mathbf{Y} \mathbf{S}_{i}^{H}=\left(\sum_{k=1}^{K} \mathbf{a}\left(\theta_{k}\right) \mathbf{S}_{k}+\mathbf{V}\right) \cdot \mathbf{S}_{i}^{H} \\
& =\left\|\mathbf{S}_{i}\right\|_{2}^{2} \mathbf{a}\left(\theta_{i}\right)+\sum_{k=1, k \neq i}^{K} \mathbf{a}\left(\theta_{k}\right) \mathbf{S}_{k} \mathbf{S}_{i}^{H}+\mathbf{V} \cdot \mathbf{S}_{i}^{H}
\end{aligned}
$$

Since the correlation between different FH signals is relatively low, the second and third term in (14) are considered as new noise term. Therefore, in this stage, the modified likelihood function of the filtered signal can be represented by

$$
p\left(\tilde{\mathbf{Y}}_{1}^{i} \mid \tilde{\mathbf{S}}_{i}\right) \sim \mathcal{C N}\left(\mathbf{Y} \mathbf{S}_{i}^{H} \mid \mathbf{A} \tilde{\mathbf{s}}_{i}, \sigma_{1}^{2}\right)
$$

where $\tilde{s}_{i}$ is a sparse vector denoting the spatial sparsity of the $i$-th FH signal. Combining the sparsity prior of $\tilde{s}_{i}$, the MAP estimation of this problem can be given as,

$$
\tilde{\mathbf{s}}_{\mathbf{i}}=\arg \min _{\tilde{\mathbf{s}}}\left\|\mathbf{Y S}_{i}^{H}-\mathbf{A} \tilde{\mathbf{s}}_{\mathbf{i}}\right\|+\lambda\left\|\tilde{\mathbf{s}}_{\mathbf{i}}\right\|_{1}
$$

where $\lambda$ is the regularization parameter to balance data fitting and sparsity.

Remark 2: Based on the estimation of the FH signal, the signal is projected on the estimated FH signal, respectively. The underlying rationale is that the DOA estimation can be more accurately carried out based on the projection. The DOA can be more accurately estimated, since the filtered signal is a one-sparse signal in spatial domain. FH signal Estimation Stage: Assume that the DOAs have been estimated in the above stage. Similarly, the beam-forming procedure is carried out to estimate the FH signal for the $i$-th direction as,

$$
\begin{aligned}
\tilde{\mathbf{Y}}_{2}^{i} & =\mathbf{a}\left(\hat{\theta}_{i}\right)^{H} \mathbf{Y}=\mathbf{a}^{H}\left(\hat{\theta}_{i}\right) \mathbf{A s}+\mathbf{a}^{H}\left(\hat{\theta}_{i}\right) \mathbf{V} \\
& =\mathbf{s}_{i}+\sum_{j=1, j \neq i}^{K_{s}} \mathbf{a}^{H}\left(\hat{\theta}_{i}\right) \cdot \mathbf{a}\left(\theta_{j}\right) \cdot \mathbf{s}_{j}+\mathbf{a}^{H}\left(\hat{\theta}_{i}\right) \mathbf{V} .
\end{aligned}
$$

Therefore, the modified likelihood function of the beamformed signal can be represented by,

$$
p\left(\tilde{\mathbf{Y}}_{2}^{i} \mid \mathbf{x}_{i}\right) \sim C N\left(\mathbf{Y}^{H} \mathbf{a}\left(\hat{\theta}_{i}\right) \mid \mathbf{W} \mathbf{x}_{i}, \sigma_{2}^{2}\right)
$$

where $\mathbf{x}_{i}$ is a sparse vector denoting the time-frequency coefficient of the FH signal from $i$-th direction. Combining the sparsity prior of $\tilde{x}_{i}$ in both hopping time and frequency domain, the MAP estimation of this problem can be given as,

$$
\mathbf{x}_{\mathbf{i}}=\arg \min _{\mathbf{x}_{\mathbf{i}}}\left\|\mathbf{Y}^{H} \mathbf{a}\left(\hat{\theta}_{i}\right)-\mathbf{W} \mathbf{x}_{\mathbf{i}}\right\|+\lambda_{1}\left\|\mathbf{x}_{\mathbf{i}}\right\|_{1}+\lambda_{2}\left\|\mathbf{D} \mathbf{x}_{\mathbf{i}}\right\|_{1}(19)
$$

Remark 3: Based on the estimation of the DOA, beamforming procedure is carried out before $\mathrm{FH}$ signal estimation. An immediate advantage is that this procedure will separate the FH signals, where each one can be considered to be sparser in both hopping time and frequency domain. Therefore, the proposed algorithm operates in an iterative manner as shown in Algorithm 1. It is noted that the number of sources $K_{s}$ is not required to be estimated by modelselection technique, rather it can be easily determined by thresholding residual due to sparsity, which is not discussed further for brevity.

\section{FH SignAL ESTIMATION PROBLEM}

In this section, the experimental results are presented to validate the performance of the algorithm. The regularization parameter for FH signal estimation stage is chosen to be $\lambda_{1}$ $=\lambda_{1}{ }^{*} / 10$ and $\lambda_{2}=\lambda_{2}{ }^{*} / 10$ to achieve desirable hopping time and frequency detection in the tested SNRs, where optimal $\lambda_{1}{ }^{*}$ and $\lambda_{2}{ }^{*}$ are given in [8]. The number of Monte Carlo trails is chosen to be 100 .

The hopping signals used in the following experiments are generated as follows: the first hopping component is active within the range of time index $[0: 15]$ and the carrier frequency hops from $13 \mathrm{KHz}$ to $18 \mathrm{KHz}$ within the range of time index [16:63]. The second hopping component is active within the range of time index $[0: 31]$ and the carrier frequency hops from $28 \mathrm{KHz}$ to $23 \mathrm{KHz}$ within the range of time index [32:63].

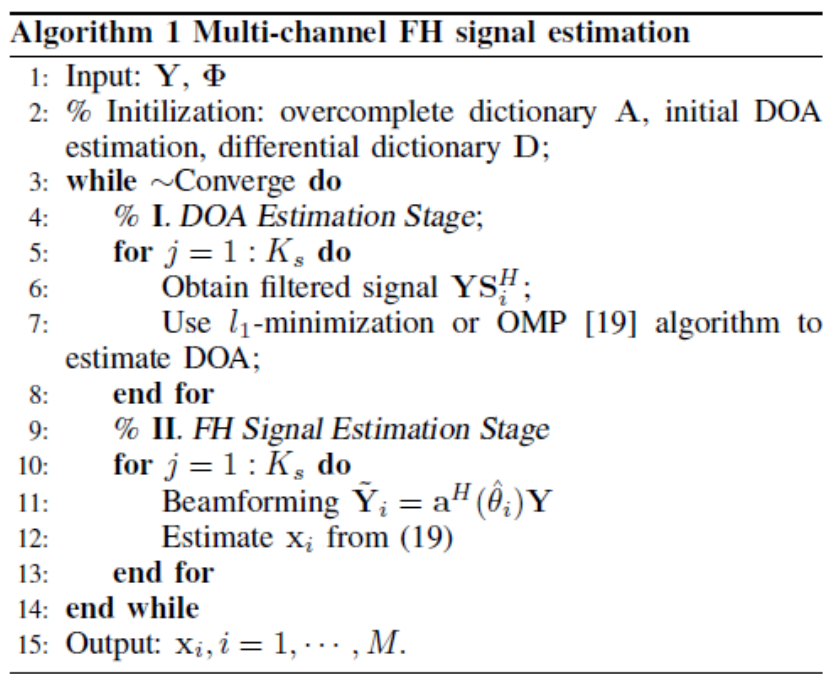

In the following, the frequency hopping signal estimation based on EM (FHSE-EM) algorithm is particularly compared for performance evaluation. In the following experiments, the SNR is defined as

$$
\mathrm{SNR}=10 \log _{10}\left(\frac{\|\mathrm{s}\|_{2}^{2}}{N_{s} \sigma^{2}}\right)
$$

where $\mathbf{s}$ denotes the signal vector, $N_{s}$ is the number of time indices and $\sigma^{2}$ is the noise power.

In particular, two performance measures are defined for comparison of hopping time and instantaneous frequency (IF) detection, respectively. The correct hopping time detection ratio is defined as,

$$
P_{t}=\left(\sum_{i=1}^{M_{c}} D_{t}(i)\right) / M_{c}
$$

where $M_{c}$ is the number of Monte Carlo trails and $D_{t}(i)$ is the number of correct detections in each Monte Carlo trial. The hopping time statistic is defined as $\Delta_{n}=\left\|x_{n+1}-x_{n}\right\|^{2}{ }_{2}$. A correct hopping time detection is declared if the estimated hopping instant is less than 3 samples away from the associated true hopping instant, which is defined in the same 
way as in [8], [18]. The incorrect IF detection ratio is further defined as,

$$
E_{f}=10 \log _{10}\left(1-\left(\sum_{i=1}^{M_{c}} D_{f}(i)\right) / M_{c}\right)
$$

where $D f(i)$ is the correct frequency detection rate in the $i$-th Monte Carlo trial. An illustrative example is given in Fig. 2. In this experiment, the true DOA of the two sources are $\boldsymbol{\theta}=[40$; 60]. It is seen that the spectrogram obtained in Fig. 2 (a) and (c) cannot give a good energy concentration of the signal, particularly in the first system dwell time. In contrast, the time frequency representation obtained by proposed sparsity-driven methods in Fig. 2 (e) and (g) can give much better estimation and concentration due to the utilization of hierarchical sparsity regularized strategy in the time-frequency domain and spatial domain, receptively. Notably, the time-frequency representation obtained by the proposed method is considered quite a desirable one since the noise is well pruned away with clear hopping time estimation compared to those obtained by
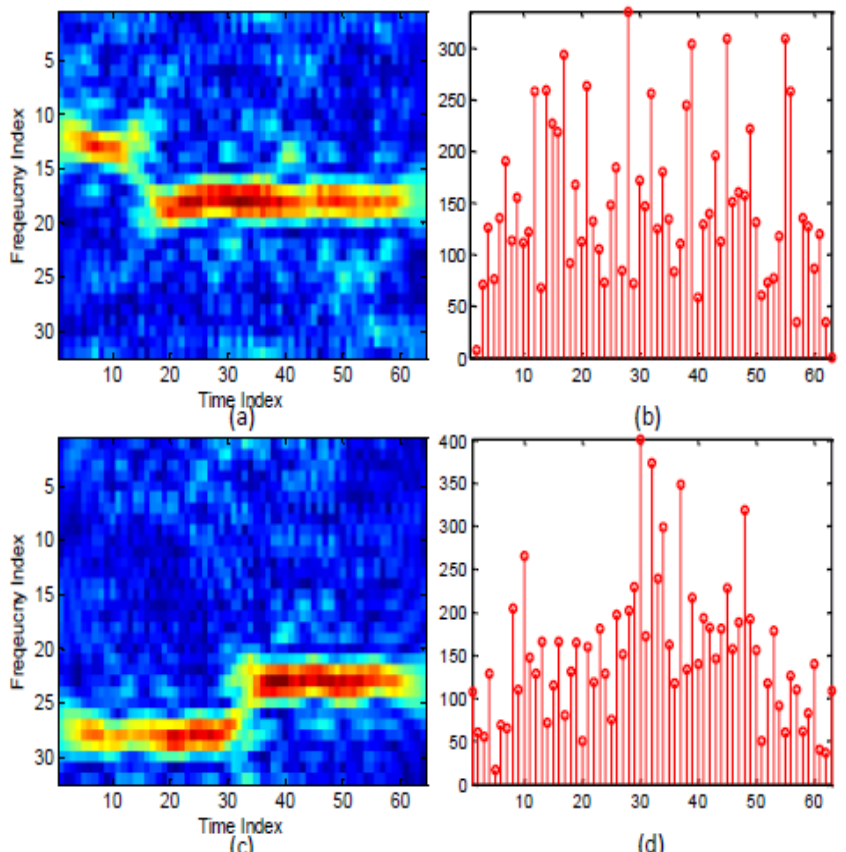

(d)
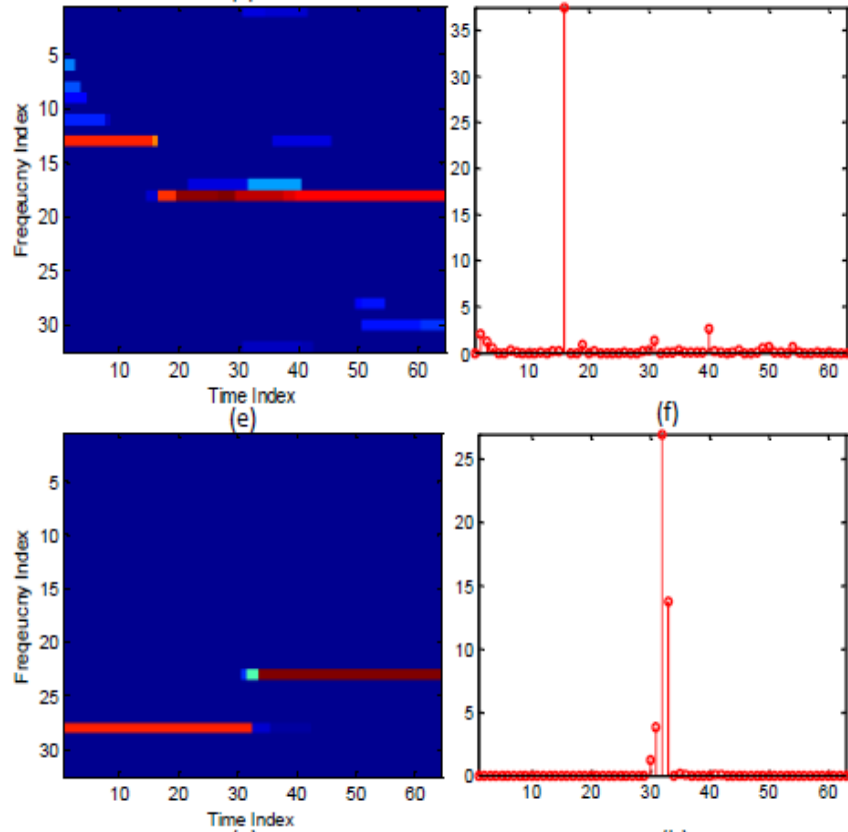

$(\mathrm{g}$

(h)

Fig. 2. An illustrative example of frequency hopping signal estimation. (a) Spectrogram (component 1) and (b) the hopping time statistic; (c) Spectrogram (component 2) and (d) the hopping time statistic; (e) proposed HSRE (component 1) and (f) the hopping time statistic; (g) proposed HSRE (component 2 ) and (h) the hopping time statistic. (The DOA estimation for STFT is $(\boldsymbol{\theta}=[38,61])$ using $\ell_{1}$-svd and DOA estimated by HSRE is $(\boldsymbol{\theta}=[40,60])$

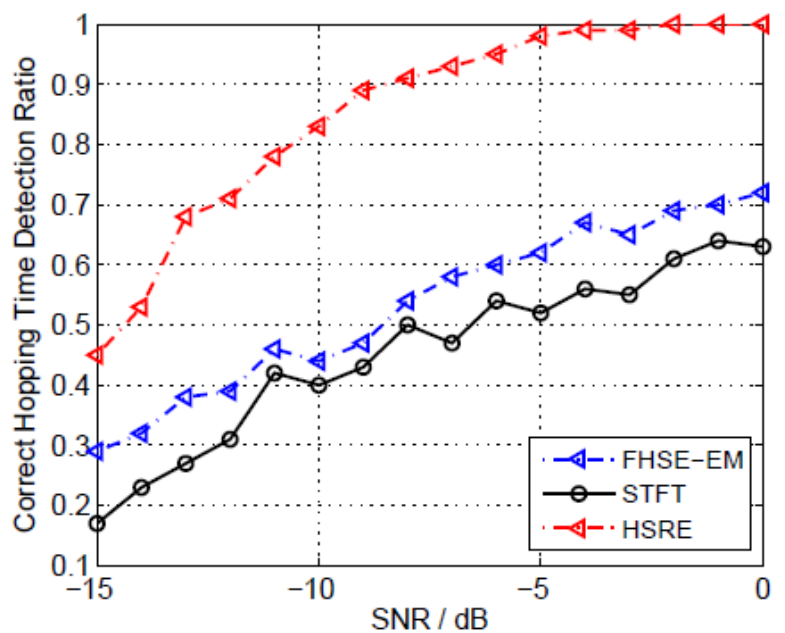

Fig. 3. The correct hopping time detection ratio for frequency hopping signal with two components is presented, in terms of SNR. The performance of the proposed approach is compared with FHSE-EM and STFT. The number of sensors is set to be 8 . 


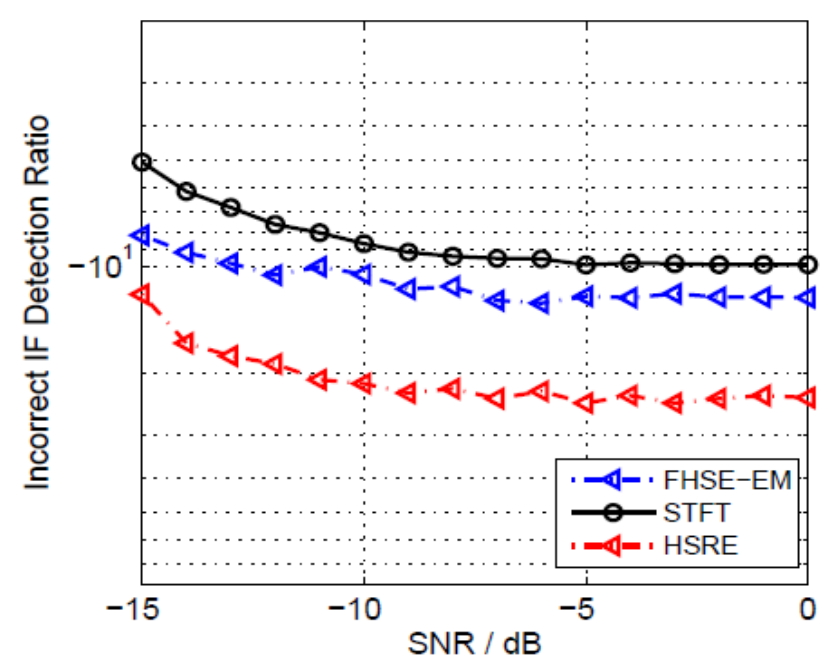

Fig. 4. The incorrect IF detection ratio for frequency hopping signal with two components is presented. The number of sensors is set to be 8 .

STFT. The corresponding hopping time statistics also demonstrate the superiority of the proposed method. As seen from Fig. 2 (b) and (d), the statistics does not give the correct and has undesirable side-lobes. In contrast, the hopping time statistics obtained by proposed algorithm, as shown in Fig. 2 (f) and (h) can give the correct estimation result and has a sharp spike in hopping time instant. Therefore, it can show that the proposed approach can achieve better DOA estimation accuracy and higher hopping time detection rate.

In Figs. 3, Monte Carlo experiments are conducted to give quantitative evaluation of the proposed algorithm and the previously reported ones, in terms of correct hopping time detection ratio. From this figure, it can be observed that with the increase of SNR, the STFT, FHSE-EM and the proposed all achieve better performance. In particular, the correct hopping time detection ratio of proposed HSRE method is almost $100 \%$, when SNR > $-5 \mathrm{~dB}$. Across all the SNRs, our proposed algorithm can achieve the best hopping time detection ratios due to the utilization of hierarchical sparsity.

In Fig. 4, the corresponding incorrect IF detection ratio is presented with same experimental settings. From this figure, similarly, all of these algorithms gives lower incorrect IF detection ratio with the increase of SNRs. In particular, our proposed algorithm can achieve the lowest incorrect IF detection ratio due to the inherent scheme of joint sparsity inducing procedure.

\section{CONCLUSION}

In this paper, a new frequency hopping signal estimation method in sensor array system is developed, where DOA, hopping time and frequency can be jointly estimated. Compared with existing research, in our work, a hierarchical framework is particularly utilized to combat the difficulties of model order selection procedure, by exploiting spatial sparsity and time-frequency sparsity. This novel approach can not only avoid the tedious parameter tuning process, but also provide robust performance in low SNR environments.

\section{REFERENCES}

[1] X. Liu, N. D. Sidiropoulos, and A. Swami, "Joint hop timing and frequency estimation for collision resolution in FH networks," IEEE Transactions on Wireless Communications, vol. 4, no. 6, pp. 3063-3074, 2005.

[2] C. Chen and P. Vaidyanathan, "MIMO radar ambiguity properties and optimization using frequency-hopping waveforms," IEEE Transactionson Signal Processing, vol. 56, no. 12, pp. 5926-5936, 2008.

[3] S. Srinivasa and S. A. Jafar, "Cognitive radios for dynamic spectrum access-the throughput potential of cognitive radio: A theoretical perspective,” IEEE Communications Magazine, vol. 45, no. 5, pp. 7379, 2007.

[4] D. J. Torrieri, "Mobile frequency-hopping CDMA systems," IEEE Transactions on Communications, vol. 48, no. 8, pp. 1318-1327, 2000.

[5] L. Zhang, H.Wang, and T. Li, "Anti-jamming message-driven frequency hopping - part I: System design," IEEE Transactions on Wireless Communications, vol. 12, no. 1, pp. 70-79, 2013.

[6] L. Zhang and T. Li, “Anti-jamming message-driven frequency hopping part II: Capacity analysis under disguised jamming," IEEE Transactionson Wireless Communications, vol. 12, no. 1, pp. 80-88, 2013.

[7] X. Liu, Nicholas D. Sidiropoulos, and A. Swami, "Blind high-resolution localization and tracking of multiple frequency hopped signals," IEEE Transactions on Signal Processing, vol. 50, no. 4, pp. 889-901, 2002.

[8] D. Angelosante, G.B. Giannakis, and N.D. Sidiropoulos, "Estimating multiple frequency-hopping signal parameters via sparse linear regression," IEEE Transactions on Signal Processing, vol. 58, no. 10, pp. 5044-5056, 2010

[9] L. Cohen, "Time-frequency distributions-a review," Proceedings of the IEEE, vol. 77, no. 7, pp. 941-981, 1989.

[10] S. Barbarossa and A. Scaglione, "Parameter estimation of spread spectrum frequency-hopping signals using time-frequency distributions," in First IEEE Signal Processing Workshop on Signal Processing Advances in Wireless Communications, 1997, pp. 213-216.

[11] X. Liu, J. Li, and X. Ma, "An EM algorithm for blind hop timing estimation of multiple FH signals using an array system with bandwidth mismatch," IEEE Transactions on Vehicular Technology, vol. 56, no. 5, pp. 2545-2554, 2007.

[12] D. Angelosante, G.B. Giannakis, and N.D. Sidiropoulos, "Sparse parametric models for robust nonstationary signal analysis: Leveraging the power of sparse regression," IEEE Signal Processing Magazine, vol. 30, no. 6, pp. 64-73, 2013.

[13] S. S. Chen, D. L. Donoho, and M.A. Saunders, "Atomic decomposition by basis pursuit," SIAM Review, vol. 43, no. 1, pp. 129-159, Jan. 2001.

[14] D. Malioutov, M. C, etin, and A. S. Willsky, "A sparse signal reconstruction perspective for source localization with sensor arrays," IEEE Transactions on Signal Processing, vol. 53, no. 8, pp. 3010-3022, 2005 .

[15] Z. Liu, Z. Huang, and Y. Zhou, "Sparsity-inducing direction finding for narrowband and wideband signals based on array covariance vectors," IEEE Transactions on Wireless Communications, vol. 12, no. 8, pp. 112, August 2013.

[16] R. Tibshirani, M. Saunders, S. Rosset, J. Zhu, and K. Knight, "Sparsity and smoothness via the fused LASSO," Journal of the Royal Statistical Society: Series B (Statistical Methodology), vol. 67, no. 1, pp. 91-108, 2005 .

[17] L. Zhao, G. Bi, L. Wang, and H. Zhang, "An improved auto-calibration algorithm based on sparse Bayesian learning framework," IEEE Signal Processing Letters, vol. 20, no. 9, pp. 889-892, 2013.

[18] L. Zhao, L. Wang, G. Bi, L. Zhang, and H. Zhang, "Robust frequencyhopping spectrum estimation based on sparse bayesian method," toappear in IEEE Transactions on Wireless Communications.

[19] E. J. Candes and M. B. Wakin, "An introduction to compressive sampling," IEEE Signal Processing Magazine, vol. 25, no. 2, pp. 21-30, Mar. 2008. 